\title{
Tighter Approximation Bounds for Minimum CDS in Unit Disk Graphs
}

\author{
Minming Li · Peng-Jun Wan · Frances Yao
}

Received: 24 March 2010 / Accepted: 1 April 2011 / Published online: 15 April 2011

(C) Springer Science+Business Media, LLC 2011

\begin{abstract}
Connected dominating set (CDS) in unit disk graphs has a wide range of applications in wireless ad hoc networks. A number of approximation algorithms for constructing a small CDS in unit disk graphs have been proposed in the literature. The majority of these algorithms follow a general two-phased approach. The first phase constructs a dominating set, and the second phase selects additional nodes to interconnect the nodes in the dominating set. In the performance analyses of these two-phased algorithms, the relation between the independence number $\alpha$ and the connected domination number $\gamma_{c}$ of a unit-disk graph plays the key role. The best-known relation between them is $\alpha \leq 3 \frac{2}{3} \gamma_{c}+1$. In this paper, we prove that $\alpha \leq 3.4306 \gamma_{c}+4.8185$. This relation leads to tighter upper bounds on the approximation ratios of two approximation algorithms proposed in the literature.
\end{abstract}

Keywords Wireless ad hoc networks · Connected dominating set · Approximation algorithm $\cdot$ Geometric analysis

\section{Introduction}

Connected dominating set (CDS) has a wide range of applications in wireless ad hoc networks (cf. a recent survey [3] and references therein). Consider a wireless ad hoc network with undirected communication topology $G=(V, E)$. A CDS of $G$ is

\footnotetext{
M. Li $(\varangle) \cdot$ F. Yao

Department of Computer Science, City University of Hong Kong, Hong Kong, Hong Kong SAR e-mail:minmli@cs.cityu.edu.hk

F. Yao

e-mail: csfyao@cityu.edu.hk

P.-J. Wan

Department of Computer Science, Illinois Institute of Technology, Chicago, USA

e-mail:wan@cs.iit.edu 
a subset $U \subset V$ satisfying that each node in $V \backslash U$ is adjacent to at least one node in $U$ and the subgraph of $G$ induced by $U$ is connected. A number of distributed algorithms for constructing a small CDS in wireless ad hoc networks have been proposed in the literature. The majority of these distributed algorithms follow a general two-phased approach [1, 2, 4, 7, 9-11]. The first phase constructs a dominating set, and the nodes in the dominating set are called dominators. The second phase selects additional nodes, called connectors, which together with the dominators induce a connected topology. The algorithms in $[1,2,4,7,9,10]$ differ in how to select the dominators and connectors. For example, the algorithm in [2] selects the dominators using the Chvatal's greedy algorithm [5] for Set Cover, the algorithms in [1,9] select an arbitrary maximal independent set (MIS) as the dominating set, and all the algorithms in $[4,7,10,11]$ choose a special MIS with 2-hop separation property as the dominating set.

The approximation ratios of these two-phased algorithms $[1,2,4,7,9,10]$ have been analyzed when the communication topology is a unit-disk graph (UDG). For a wireless ad hoc network in which all nodes lie in a plane and have equal maximum transmission radii normalized to one, its communication topology $G=(V, E)$ is often modelled by a UDG in which there is an edge between two nodes if and only if their Euclidean distance is at most one. Except the algorithms in [2,9] which have logarithmic and linear approximations ratios respectively, all other algorithms in $[1,4,7,10,11]$ have constant approximation ratios. The algorithm in [1] targets at distributed construction of CDS in linear time and linear messages. With this objective, it trades the size of the CDS with the time complexity, and thus its approximation ratio is a large constant (but less than 192). The analyses of the algorithms in $[4,7,10,11]$ rely on the relation between the independence number (the size of a maximum independent set) $\alpha$ and the connected domination number (the size of a minimum connected dominating set) $\gamma_{c}$ of a connected UDG $G$. A loose relation $\alpha \leq 4 \gamma_{c}+1$ was obtained in [10], which implies an upper bound of 8 on the approximation ratios of both algorithms in $[4,10]$. A refined relation $\alpha \leq 3.8 \gamma_{c}+1.2$ was discovered in [12]. With such a refined relation, the upper bound on the approximation ratios of both algorithms in $[4,10]$ was reduced from 8 to 7.6 , and an upper bound of $5.8+\ln 5 \approx 7.41$ on the approximation ratio of the algorithms in [7] was derived (the bound $4.8+\ln 5 \approx 6.41$ in [7] was incorrect). The best-known relation $\alpha \leq 3 \frac{2}{3} \gamma_{c}+1$ if $G$ has at least two nodes was recently proven in [11]. As a result, the upper bound on the approximation ratio of the algorithm in [10] was further reduced to $7 \frac{1}{3}$ in [11]. Another greedy approximation algorithm was also proposed in [11] and its approximation ratio was proven to be bounded by $6 \frac{7}{18}$.

In this paper, we first prove a further improved relation $\alpha \leq 3.4306 \gamma_{c}+4.8185$ in Sect. 3. The proof for this bound employs an integrated area and length argument, and involves some other interesting extreme geometric problems studied in Sect. 2. Subsequently in Sect. 4, we provide tighter analyses of the approximation algorithm in [10] and the other greedy algorithm in [11]. We prove that the approximation ratio of the former algorithm is at most 6.862 and the approximation ratio of the latter algorithm is at most 6.075 .

We remark that a recent paper [6] claimed that for any connected UDG $G$,

$$
\alpha \leq 3.453 \gamma_{c}+8.291 \text {. }
$$


However, as discovered in [11], the proof for a key geometric extreme property underlying such claim was missing, and such proof is far from being apparent or easy. Such property is rigorously proved in Lemma 6. The proof for Lemma 6 is quite lengthy and delicate. Indeed, the whole Sect. 2 is part of this proof. Consequently, the bound claimed in [6] can be treated at most as a conjecture at the time of its publication rather than a proven result.

In the remaining of this section, we introduce some terms and notations. For any point $u$ and any $r>0$, we use $\operatorname{disk}_{r}(u)$ to denote the closed disk of radius $r$ centered at $u$, and circle $_{r}(u)$ to denote the boundary circle of $\operatorname{disk}_{r}(u)$. A path or a polygon is said to be inscribed in a circle if all its vertices lie on the circle. The Lebesgue measure (or area) of a measurable set $A \subset \mathbb{R}^{2}$ is denoted by $|A|$. The topological boundary of a set $A \subset \mathbb{R}^{2}$ is denoted by $\partial A$. For the simplicity of presentation, the line segment between two points $u$ and $v$ and its length are both denoted by $u v$ by slightly abusing the notation, but the actual meaning can be clearly told from the context.

\section{A Geometric Extreme of Polygon}

The technical approach to deriving improved relation between $\alpha$ and $\gamma_{c}$ is an integrated area and length argument. In this section, we present some area extremes of polygons which will be used intensively in the area argument to be given in Sect. 3 .

Suppose that $s, o, t$ and $t^{\prime}$ are four points from the left to the right on a horizontal line with $o s=1$, ot $=0.5$ and $o t^{\prime}=1 / \sqrt{3}$. If $P$ is a regular hexagon centered at $o$ with $t^{\prime}$ as a vertex, it is easy to compute that $|P|=\sqrt{3} / 2$ and $\left|P \cap \operatorname{disk}_{1.5}(s)\right|=\sigma$, where $\sigma$ is the constant

$$
\frac{\sqrt{3}}{6}-\frac{1}{2}+\frac{\sqrt{8+2 \sqrt{3}}}{4}+\frac{3 \pi}{8}-\frac{9}{4} \arccos \frac{1+\sqrt{3}}{3} \approx 0.85505328 .
$$

For an arbitrary polygon $P$ which is inscribed in circle $_{1 / \sqrt{3}}(o)$ and contains $\operatorname{dis}_{0.5}(\mathrm{o})$, it has the following geometric extreme property.

Theorem 1 Suppose that $P$ is a polygon inscribed in circle ${ }_{1 / \sqrt{3}}(o)$ satisfying that $\operatorname{disk}_{0.5}(o) \subseteq P$. Then,

$$
\begin{aligned}
& |P| \geq \sqrt{3} / 2, \\
& \left|P \cap \operatorname{disk}_{1.5}(s)\right| \geq \sigma .
\end{aligned}
$$

The first inequality in Theorem 1 can be easily proved by using the property of the sine function.

Lemma 1 Suppose that $\phi>0$. Then, for any set of angles $\left\{\theta_{i}: 1 \leq i \leq k\right\}$ satisfying that $\sum_{i=1}^{k} \theta_{i}=\phi$ and $0<\theta_{i} \leq \frac{\pi}{3}$, we have

$$
\sum_{i=1}^{k} \sin \theta_{i} \geq\left\lfloor\frac{\phi}{\frac{\pi}{3}}\right\rfloor \sin \frac{\pi}{3}+\sin \left(\phi-\left\lfloor\frac{\phi}{\frac{\pi}{3}}\right\rfloor \frac{\pi}{3}\right) .
$$


Proof Suppose that $S=\left\{\theta_{i}: 1 \leq i \leq k\right\}$ is a set of angles satisfying that $\sum_{i=1}^{k} \theta_{i}=\phi$, $0<\theta_{i} \leq \frac{\pi}{3}$ and $\sum_{i=1}^{k} \sin \theta_{i}$ achieves the minimum. We first claim that the sum of any pair of angles in $S$ is greater than $\frac{\pi}{3}$. Assume to the contrary that there are two angles $\theta_{1}$ and $\theta_{2}$ in $S$ with $\theta_{1}+\theta_{2} \leq \frac{\pi}{3}$. Replacing the two angles $\theta_{1}$ and $\theta_{2}$ by the single angle $\theta_{1}+\theta_{2}$ would strictly decrease the total sine values of the angles in $S$. This is a contradiction, and hence our first claim holds.

Secondly, we claim that at most one angle in $S$ is less than $\frac{\pi}{3}$. Assume to the contrary that there are two angles $\theta_{1}$ and $\theta_{2}$ in $S$ which are less than $\frac{\pi}{3}$. By symmetry, we assume that $\theta_{1} \leq \theta_{2}$. Then $0<\theta_{1}+\theta_{2}-\frac{\pi}{3} \leq \theta_{1} \leq \theta_{2}<\frac{\pi}{3}$. Since the sine function is concave over $\left[0, \frac{\pi}{3}\right]$ and $\theta_{1}-\left(\theta_{1}+\theta_{2}-\frac{\pi}{3}\right)=\frac{\pi}{3}-\theta_{2}$, we have

$$
\sin \frac{\pi}{3}-\sin \theta_{2}<\sin \theta_{1}-\sin \left(\theta_{1}+\theta_{2}-\frac{\pi}{3}\right) \text {. }
$$

Hence,

$$
\sin \theta_{1}+\sin \theta_{2}>\sin \left(\theta_{1}+\theta_{2}-\frac{\pi}{3}\right)+\sin \frac{\pi}{3} .
$$

So, replacing $\theta_{1}$ and $\theta_{2}$ by $\theta_{1}+\theta_{2}-\frac{\pi}{3}$ and $\frac{\pi}{3}$ would strictly decrease the total sine values of the angles in $S$. This is a contradiction, and hence our second claim holds.

Therefore, $S$ must consist of $\left\lceil\frac{\phi}{\frac{\pi}{3}}\right\rceil$ angles, among which $\left\lfloor\frac{\phi}{\frac{\pi}{3}}\right\rfloor$ angles are equal to $\frac{\pi}{3}$. So, the lemma holds.

Lemma 1 implies that for any polygon $P$ inscribed in circle $_{1 / \sqrt{3}}(o)$ satisfying that circle $_{0.5}(o) \subseteq P$, we have

$$
|P| \geq 6 \cdot \frac{1}{2}\left(\frac{1}{\sqrt{3}}\right)^{2} \sin \frac{\pi}{3}=\frac{\sqrt{3}}{2} .
$$

Thus, the first inequality in Theorem 1 holds.

In the next, we prove the second inequality in Theorem 1. We first introduce a special type of polygons called canonical polygons. For any pair of points $u$ and $v$ on circle $_{1 / \sqrt{3}}(o)$, let $\widehat{u v}$ be the arc in circle $_{1 / \sqrt{3}}(o)$ from $u$ to $v$ in the counterclockwise manner. Denote by $\phi$ the radian of $\widehat{u v}$, and let $k=\lceil\phi /(\pi / 3)\rceil$. We construct a path $Q$ of $k$ edges from $u$ to $v$ with all vertices on $\widehat{u v}$ as follows: If $\phi$ is an integer multiple of $\frac{\pi}{3}$, then all edges of $Q$ are tangent to circle $_{0.5}(o)$; otherwise, all edges except the $\lceil k / 2\rceil$-th edge are tangent to circle $_{1 / \sqrt{3}}(o)$ (we remark that in this case, the $\lceil k / 2\rceil$-th edge is disjoint from circle $\left.e_{1 / \sqrt{3}}(o)\right)$. The path $Q$ is referred to as the canonical path inscribed in circle $_{1 / \sqrt{3}}(o)$ from $u$ to $v$. For any point $u$ which lies on the right side the vertical line through $t$, we construct a polygon $P$ as follows: let $u_{1}$ and $u_{2}$ be the two points on circle $e_{1 / \sqrt{3}}(o)$ such that the two line segments $u_{1} u$ and $u_{2} u$ are tangent to circle $_{1 / \sqrt{3}}(o)$ and $u_{1}$ is above the line st. Then, $P$ is surrounded by $u_{1} u, u_{2} u$ and the canonical path from $u_{1}$ to $u_{2}$. The polygon $P$ is referred to as the canonical polygon of $u$. The point $u$ is called the base vertex of $P$, and the angle $\theta=\arccos \frac{1}{2 o u}$ is called the base angle of $P$. Note that if $u$ is on the ray ot, then $P$ is symmetric with respect to the line $o t$, and the area of $P \cap \operatorname{disk}_{1.5}(s)$ is a function of the base angle $\theta$, which is denoted by $f(\theta)$. Note that $f\left(\frac{\pi}{6}\right)=\sigma$. We will derive the explicit expression of 
Fig. 1 Calculation of $\angle w s t$ and $g(\theta)$

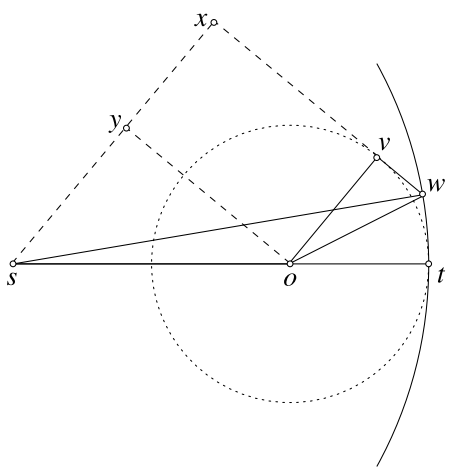

$f(\theta)$ and explore some useful properties of the function $f(\theta)$. We will also prove that for any canonical polygon $P,\left|P \cap \operatorname{disk}_{1.5}(s)\right| \geq f(\theta)$ where $\theta$ is the base angle of $P$.

We define a geometric function $g$ on $[0, \pi]$ as follows. For any $\theta \in[0, \pi]$, let $v$ be a point on circle $_{1 / 2}(o)$ satisfying that $\angle t o v=\theta$ and $v$ is above st. Let $w$ be the point on circle $1 / \sqrt{3}(o)$ satisfying that $v w$ is tangent to circle $_{1 / 2}(o)$ and $w$ lies to the right of $v$. Then, $g(\theta)$ is defined to be the area of the region surrounded by arc $t w$ and the three line segments ot, ov and $v w$ (see Fig. 1). The next lemma presents the explicit expressions of $g(\theta)$ and its first and second order derivatives.

Lemma 2 Let $\beta=\theta-\arccos \frac{1+2 \cos \theta}{3}$. Then,

$$
\begin{aligned}
g(\theta) & =\frac{9}{8} \beta-\frac{3}{4} \sin \beta+\frac{\sqrt{6}}{4} \sin \frac{\beta}{2}, \\
g^{\prime}(\theta) & =\frac{13}{8}-1.5 \cos \beta \\
g^{\prime \prime}(\theta) & =1.5\left(1-\sqrt{1-\frac{1}{2+\cos \theta}}\right) \sin \beta .
\end{aligned}
$$

In addition, $g$ is increasing and convex on $[0, \pi]$, while both $g^{\prime}$ and $g^{\prime \prime}$ are increasing on $\left[0, \frac{\pi}{2}\right]$.

Proof We first show that $\angle w s t=\beta$. Let $x$ be the perpendicular foot of $s$ in the line $v w$, and $y$ be the perpendicular foot of $o$ in $s x$ (see Fig. 1). Then, $\angle o s y=\theta$ and $y x=o v=0.5$. So

$$
s x=s y+y x=0.5+\cos \theta .
$$

Hence,

$$
\angle w s x=\arccos \frac{1+2 \cos \theta}{3} .
$$


Fig. 2 Geometric proof for $g^{\prime}(\theta)=\frac{o w^{2}}{2}$

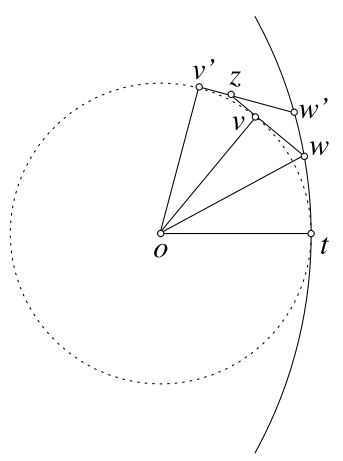

Therefore,

$$
\angle w s t=\angle t s x-\angle w s x=\theta-\arccos \frac{1+2 \cos \theta}{3}=\beta .
$$

Now, we derive the expression of $g(\theta)$. By applying law of cosines to $\triangle o s w$, $o w^{2}=13 / 4-3 \cos \beta$. Hence,

$$
v w=\sqrt{o w^{2}-1 / 4}=\sqrt{6} \sin \frac{\beta}{2} .
$$

Since $g(\theta)$ is equal to the area of the sector stw minus the area of $\triangle o s w$ and then plus the area of $\triangle o v w$, we have

$$
g(\theta)=\frac{9}{8} \beta-\frac{3}{4} \sin \beta+\frac{1}{4} v w=\frac{9}{8} \beta-\frac{3}{4} \sin \beta+\frac{\sqrt{6}}{4} \sin \frac{\beta}{2} .
$$

Next, we take a geometric approach to compute $g^{\prime}(\theta)$. We rotate $v$ counterclockwise by a small angle $\delta$ to a point $v^{\prime}$ (see Fig. 2). Then the point $w$ moves along circle $_{1.5}(s)$ to a new point denoted by $w^{\prime}$. Denote by $z$ the intersection point between the two lines tangent to $\operatorname{circle}_{0.5}(o)$ at $v$ and $v^{\prime}$ respectively. Let $\Delta_{1}, \Delta_{2}$ and $\Delta_{3}$ denote the areas of the quadruple $o v z v^{\prime}, \Delta z w w^{\prime}$, and the circular segment subtended by $w w^{\prime}$ respectively. Then, $g(\theta+\delta)-g(\theta)=\Delta_{1}+\Delta_{2}+\Delta_{3}$. Clearly, $\Delta_{1}=$ $\frac{1}{2} v z=\frac{1}{4} \tan \frac{\delta}{2}$. Hence, $\lim _{\delta \rightarrow 0} \frac{\Delta_{1}}{\delta}=\frac{1}{8}$. Since $\angle w z w^{\prime}=\delta, \Delta_{2}=\frac{1}{2} z w \cdot z w^{\prime} \cdot \sin \delta$. As $\delta \rightarrow 0$, both $z w$ and $z w^{\prime}$ converge to $v w$ while $\frac{\sin \delta}{\delta}$ converges to 1 , and hence $\lim _{\delta \rightarrow 0} \frac{\Delta_{2}}{\delta}=\frac{1}{2} v w^{2}$. Now,

$$
\Delta_{3}=\frac{9}{8}\left(\angle w s w^{\prime}-\sin \angle w s w^{\prime}\right)=\frac{9}{8} \angle w s w^{\prime}\left(1-\frac{\sin \angle w s w^{\prime}}{\angle w s w^{\prime}}\right) .
$$

As $\delta \rightarrow 0, \angle w s w^{\prime} \rightarrow 0$ as well, and $\frac{\angle w s w^{\prime}}{\delta} \rightarrow \frac{d \beta}{d \theta}$ which is bounded for any given $\theta$. Thus,

$$
\lim _{\delta \rightarrow 0} \frac{\Delta_{3}}{\delta}=\frac{9}{8} \frac{d \beta}{d \theta} \cdot \lim _{\delta \rightarrow 0}\left(1-\frac{\sin \angle w s w^{\prime}}{\angle w s w^{\prime}}\right)=\frac{9}{8} \frac{d \beta}{d \theta} \cdot(1-1)=0
$$


Fig. 3 The curve of $f$ on $\left[0^{\circ}, 90^{\circ}\right)$

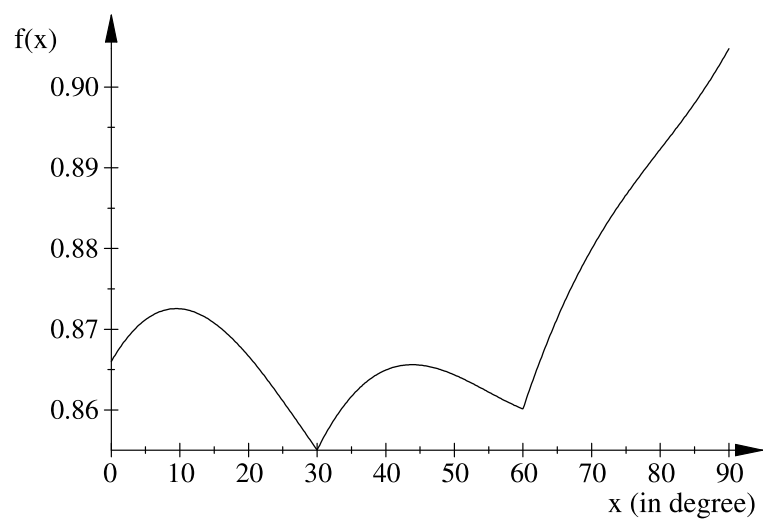

Therefore,

$$
g^{\prime}(\theta)=\lim _{\delta \rightarrow 0} \frac{\Delta_{1}+\Delta_{2}+\Delta_{3}}{\delta}=\frac{1 / 4+v w^{2}}{2}=\frac{o w^{2}}{2}=\frac{13}{8}-1.5 \cos \beta .
$$

A straightforward calculation yields

$$
\frac{d \beta}{d \theta}=1-\sqrt{\frac{1+\cos \theta}{2+\cos \theta}}=1-\sqrt{1-\frac{1}{2+\cos \theta}} .
$$

Thus,

$$
g^{\prime \prime}(\theta)=1.5 \frac{d \beta}{d \theta} \sin \beta=1.5\left(1-\sqrt{1-\frac{1}{2+\cos \theta}}\right) \sin \beta .
$$

Since both $g^{\prime}$ and $g^{\prime \prime}$ are non-negative on $[0, \pi], g$ is increasing and convex on $[0, \pi]$ while $g^{\prime}$ is increasing on $[0, \pi]$. Clearly, $\frac{d \beta}{d \theta}$ is positive and increasing with $\theta$ on $[0, \pi]$, and consequently $\beta$ is also strictly increasing with $\theta$ on $[0, \pi]$. When $\theta \in\left[0, \frac{\pi}{2}\right], \beta$ is acute, and hence $g^{\prime \prime}$ is increasing on $\left[0, \frac{\pi}{2}\right]$.

It is easy to show that $f(\theta)=2 g(\theta)+h(\theta)$, where

$$
h(\theta)=\frac{1}{4 \sqrt{3}}\left\lfloor 6-\frac{\theta}{\frac{\pi}{6}}\right\rfloor+\frac{1}{6} \sin 2\left(\left(6-\left\lfloor 6-\frac{\theta}{\frac{\pi}{6}}\right\rfloor\right) \frac{\pi}{6}-\theta\right) .
$$

Figure 3 is the curve of $f$ on $\left[0^{\circ}, 90^{\circ}\right)$. We observe and will prove later that $f$ is increasing on $\left[60^{\circ}, 90^{\circ}\right)$. However, on either of the two intervals $\left[0^{\circ}, 30^{\circ}\right]$ and $\left[30^{\circ}, 60^{\circ}\right], f$ is neither monotone, nor concave, and nor convex. Fortunately, on either of these two intervals $f$ has the following weak but still nice quasi-concave property: $f$ is said to be quasi-concave on an interval $[a, b] \subset\left[0^{\circ}, 90^{\circ}\right)$ if for each triple of increasing values $\theta_{1}, \theta_{2}, \theta_{3}$ in $[a, b], f\left(\theta_{2}\right) \geq \min \left\{f\left(\theta_{1}\right), f\left(\theta_{3}\right)\right\}$.

Lemma $3 f$ is quasi-concave on $\left[0, \frac{\pi}{6}\right]$ and $\left[\frac{\pi}{6}, \frac{\pi}{3}\right]$ respectively, and increasing on $\left[\frac{\pi}{3}, \frac{\pi}{2}\right)$. 
Proof We first show that $f$ is concave on $\left[0, \frac{3 \pi}{20}\right]$ and decreasing on $\left[\frac{3 \pi}{20}, \frac{\pi}{6}\right]$. Note that on $\left[0, \frac{\pi}{6}\right]$,

$$
\begin{aligned}
h^{\prime}(\theta) & =-\frac{1}{3} \cos 2\left(\frac{\pi}{6}-\theta\right), \\
h^{\prime \prime}(\theta) & =-\frac{2}{3} \sin 2\left(\frac{\pi}{6}-\theta\right) .
\end{aligned}
$$

Clearly, $h^{\prime \prime}$ is increasing on $\left[0, \frac{\pi}{6}\right]$. Thus, $f^{\prime \prime}$ is also increasing on $\left[0, \frac{\pi}{6}\right]$. For any $\theta \in\left[0, \frac{3 \pi}{20}\right]$,

$$
f^{\prime \prime}(\theta) \leq f^{\prime \prime}\left(\frac{3}{20} \pi\right)<0 .
$$

So, $f$ is concave on $\left[0, \frac{3 \pi}{20}\right]$. For any $\theta \in\left[\frac{3 \pi}{20}, \frac{\pi}{6}\right]$,

$$
f^{\prime}(\theta) \leq 2 g^{\prime}(\pi / 6)+h^{\prime}\left(\frac{3}{20} \pi\right)<0 .
$$

So, $f$ is decreasing over $\left[\frac{3 \pi}{20}, \frac{\pi}{6}\right]$.

Now, we show that $f$ is quasi-concave over $\left[0, \frac{\pi}{6}\right]$. Consider any $0 \leq \theta_{1} \leq \theta_{2} \leq$ $\theta_{3} \leq \frac{\pi}{6}$. If $\theta_{3} \leq \frac{3 \pi}{20}$, then $f\left(\theta_{2}\right) \geq \min \left\{f\left(\theta_{1}\right), f\left(\theta_{3}\right)\right\}$ due to the concavity of $f$ over $\left[0, \frac{3 \pi}{20}\right]$. If $\theta_{2} \geq \frac{3 \pi}{20}$, then $f\left(\theta_{2}\right) \geq f\left(\theta_{3}\right)$ since $f$ is decreasing over $\left[\frac{3 \pi}{20}, \frac{\pi}{6}\right]$. If $\theta_{2}<$ $\frac{3 \pi}{20}<\theta_{3}$, then $f\left(\theta_{3}\right) \leq f\left(\frac{3 \pi}{20}\right)$ and $f\left(\theta_{2}\right) \geq \min \left\{f\left(\theta_{1}\right), f\left(\frac{3 \pi}{20}\right)\right\}$, which together imply that $f\left(\theta_{2}\right) \geq \min \left\{f\left(\theta_{1}\right), f\left(\theta_{3}\right)\right\}$.

By a similar argument, we can prove that $f$ is concave over $\left[\frac{\pi}{6}, \frac{3 \pi}{10}\right]$ and decreasing over $\left[\frac{3 \pi}{10}, \frac{\pi}{3}\right]$. Therefore, for any $\frac{\pi}{6} \leq \theta_{1} \leq \theta_{2} \leq \theta_{3} \leq \frac{\pi}{3}, f\left(\theta_{2}\right) \geq \min \left\{f\left(\theta_{1}\right), f\left(\theta_{3}\right)\right\}$.

Next, we prove that $f$ is increasing over $\left[\frac{\pi}{3}, \frac{\pi}{2}\right)$ by showing that $f^{\prime}$ is positive over $\left[\frac{\pi}{3}, \frac{\pi}{2}\right)$. Note that $h^{\prime}(\theta)=\frac{1}{3} \cos 2 \theta$ for $\theta \in\left[\frac{\pi}{3}, \frac{\pi}{2}\right)$. For any $\theta \in\left[\frac{\pi}{3}, \frac{5 \pi}{12}\right]$,

$$
f^{\prime}(\theta) \geq 2 g^{\prime}(\pi / 3)+h^{\prime}\left(\frac{5}{12} \pi\right)>0 \text {. }
$$

For any $\theta \in\left[\frac{5 \pi}{12}, \frac{\pi}{2}\right)$,

$$
f^{\prime}(\theta) \geq 2 g^{\prime}\left(\frac{5}{12} \pi\right)-\frac{1}{3}>0 .
$$

It is easy to verify that $f(0)=\sqrt{3} / 2, f\left(\frac{\pi}{6}\right)=\sigma$, and

$$
f\left(32^{\circ}\right)<f\left(\frac{\pi}{3}\right)<f\left(34^{\circ}\right) .
$$

So, by Lemma 3 we have the following corollary.

Corollary 1 The minimum of $f$ on the interval $\left[0^{\circ}, 90^{\circ}\right)$ (respectively, $\left[32^{\circ}, 90^{\circ}\right)$ and $\left[34^{\circ}, 90^{\circ}\right)$ ) is achieved at $30^{\circ}$ (respectively, $32^{\circ}$ and $60^{\circ}$ ). 
Next, we prove the following extreme property of the canonical polygons.

Lemma 4 For any canonical polygon $P$ with base angle $\theta,\left|P \cap \operatorname{disk}_{1.5}(s)\right| \geq f(\theta)$.

Proof Denote by $u$ the base vertex of $P$, and let $v_{1}$ and $v_{2}$ be the two points on circle $_{0.5}(o)$ satisfying that $u v_{1}$ and $u v_{2}$ are tangent to circle $_{0.5}(o)$. Denote by $\theta_{1}$ and $\theta_{2}$ the radians of the two arcs $\widehat{v_{1} t}$ and $\widehat{v_{2} t}$ respectively. Then $\theta_{1}+\theta_{2}=2 \theta$, and $\mid P \cap$ $\operatorname{disk}_{1.5}(s) \mid=g\left(\theta_{1}\right)+g\left(\theta_{2}\right)+h(\theta)$. By Lemma 2, $g$ is convex, and hence $g\left(\theta_{1}\right)+$ $g\left(\theta_{2}\right) \geq 2 g\left(\frac{\theta_{1}+\theta_{2}}{2}\right)=2 g(\theta)$. Thus, $\left|P \cap \operatorname{disk}_{1.5}(s)\right| \geq 2 g(\theta)+h(\theta)=f(\theta)$.

Corollary 1 and Lemma 4 imply that $\left|P \cap \operatorname{disk}_{1.5}(s)\right| \geq \sigma$ for any canonical polygon $P$. We move on to prove the same inequality holds for any polygon $P$ inscribed in circle $_{1 / \sqrt{3}}(o)$ satisfying that circle $_{0.5}(o) \subseteq P$. All vertices of any such polygon $P$ can be classified into two categories. A vertex of $P$ is called an inner vertex if it belongs to $\operatorname{disk}_{1.5}(s)$, and an outer vertex otherwise. Let $u_{1}$, respectively $u_{2}$, be the rightmost inner vertex of $P$ above, respectively below $s t$. By Lemma 1, replacing all the internal vertices of $P$ by the vertices of the canonical path from $u_{1}$ to $u_{2}$ would not increase $\left|P \cap \operatorname{disk}_{1.5}(s)\right|$. Thus, from now on we assume that all internal vertices of $P$ are the vertices of the canonical path from $u_{1}$ to $u_{2}$. Let $u^{\prime}$ be the point such that the two line segments $u_{1} u^{\prime}$ and $u_{2} u^{\prime}$ are both tangent to $\operatorname{circle}_{0.5}(o)$. Then, $u^{\prime}$ is on the right side of the vertical line through $t$. Let $P^{\prime}$ be the canonical polygon of $u^{\prime}$. If no side of $P$ is a secant of $\operatorname{disk}_{1.5}(s)$, then

$$
\left|P \cap \operatorname{disk}_{1.5}(s)\right| \geq\left|P^{\prime} \cap \operatorname{disk}_{1.5}(s)\right| \geq \sigma .
$$

So, we further assume that at least one side of $P$ is a secant of $\operatorname{disk}_{1.5}(s)$. We will prove that $\left|P \cap \operatorname{disk}_{1.5}(s)\right|>\sigma$.

Let $p$, respectively $p^{\prime}$, be the upper, respectively lower, intersection point between circle $_{1 / \sqrt{3}}(o)$ and circle $_{1.5}(s)$, and let $q$, respectively $q^{\prime}$, be the upper, respectively lower, intersection point between the vertical line through $t$ and circle $_{1.5}(s)$ (see Fig. 4). A straightforward calculation yields

$$
\angle p o t=\arccos \frac{11 \sqrt{3}}{24} \approx 37.453^{\circ} \text {, }
$$

Fig. 4 The basic configuration

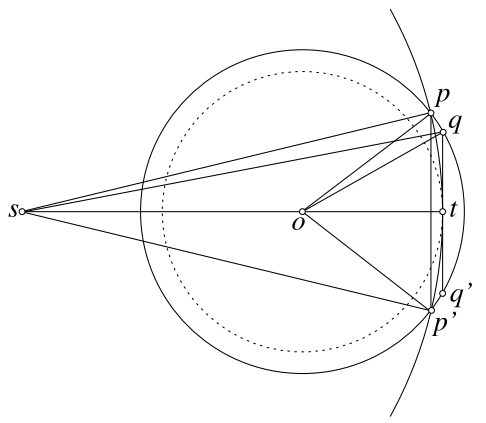




$$
\begin{aligned}
& \angle p o q=\angle p o t-\frac{\pi}{6} \approx 7.453^{\circ}, \\
& \angle o p s=\arccos \frac{19 \sqrt{3}}{36} \approx 23.916^{\circ} .
\end{aligned}
$$

Thus, any side of $P$ which is a secant of $\operatorname{circle}_{1.5}(s)$ must have its left endpoint on either the minor arc $\widehat{p q}$ or the minor arc $\widehat{p^{\prime} q^{\prime}}$, and its right endpoint on the minor arc $\widehat{q q^{\prime}}$. Consequently, there are at most two sides of $P$ which are secants of $\operatorname{circle}_{1.5}(s)$. Next, we show that at most one side of $P$ is a secant of $\operatorname{circle}_{1.5}(s)$ using the following lemma.

Lemma 5 Suppose that $e$ is a side of $P$ which is a secant of circle ${ }_{1.5}(s)$. Then, the central angle of $e$ at $o$ is greater than $2 \angle o p s$, and the central angle of the chord $e \cap \operatorname{disk}_{1.5}(s)$ at $s$ is at most $\frac{\pi}{3}-2 \angle o p s$.

Proof Let $u$ be the left endpoint of $e$ and assume by symmetry that $u$ lies on the arc $\widehat{p q}$. Denote by $a$ the chord $e \cap \operatorname{disk}_{1.5}(s)$ of circle $_{1.5}(s)$. Let $v$ be the midpoint of $e$ and $w$ be the midpoint of $a$ (see Fig. 5(a)). Then, $v$ and $w$ are the perpendicular feet of $o$ and $s$ respectively on $e, v$ lies above $s t$, and $w$ lies between $u$ and $v$ on $e$. Let $x$ be the perpendicular foot of $o$ in $s w$. Then

$$
s w=s x+x w=\cos \angle w s t+o v=\cos \angle v o t+o v=\cos (\angle u o t-\angle u o v)+o v .
$$

Suppose we fix the length of $e$ but allow $u$ to freely move along the arc $\widehat{p q}$ towards $p$. When $u$ moves towards $p$, both $\angle u o v$ and $o v$ are fixed but $\angle u o t$ increases, hence $s w$ decreases and $e$ remains as secant of $\operatorname{circle}_{1.5}(s)$. Similarly, suppose we fix $u$ but allow $e$ to increase its length. When the length of $e$ increases, both $\angle u o v$ and $o v$ decrease, hence $s w$ decreases and $e$ remains as secant of $\operatorname{circle}_{1.5}(s)$.

Now, we show that the central angle of $e$ at $o$ must be greater than $2 \angle o p s$. Assume to the contrary that the central angle of $e$ at $o$ is at most $2 \angle o p s$. We first move $u$ to $p$ while fixing the length of $e$, and then subsequently increase the central angle of $e$ to $2 \angle$ ops while fixing $u$ to the point $p$ (see Fig. 5(b)). Then $e$ would still be a secant of circle $_{1.5}(s)$. On the other hand, the central angle of $e$ is $2 \angle p o v$ in this case and hence $\angle p o v=\angle o p s$. So, $s p$ is parallel to $o v$ and is thus perpendicular to $e$. This means that

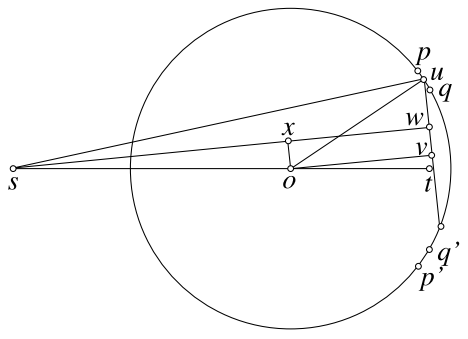

(a)

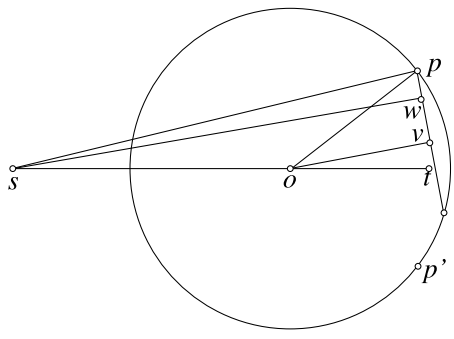

(b)

Fig. 5 A side $e$ of $P$ which is a secant of circle $_{1.5}(s)$ 
$e$ is tangent to $\operatorname{circle}_{1.5}(s)$ at $p$, which is a contradiction. Therefore, the central angle of $e$ at $o$ must be greater than $2 \angle o p s$.

Since the central angle of $a$ decreases with $s w$, it achieves its maximum when $u=p$ and the central angle of $e$ is $\frac{\pi}{3}$ (see Fig. 5(b)). In this case, the central angle of $a$ at $s$ is

$$
2 \angle p s w=2\left(\frac{\pi}{2}-\angle s p w\right)=2\left(\frac{\pi}{2}-\left(\angle o p s+\frac{\pi}{3}\right)\right)=\frac{\pi}{3}-2 \angle o p s .
$$

Thus, the central angle of $a$ at $s$ is at most $\frac{\pi}{3}-2 \angle o p s$ in general.

Since

$$
\angle p o p^{\prime}=2 \angle p o t=2(\angle o p s+\angle p s o)<2 \cdot 2 \angle o p s=4 \angle o p s .
$$

Lemma 5 implies that exactly one side of $P$ is a secant of $\operatorname{circle}_{1.5}(s)$. We denote such side by $e$ and assume by symmetry that the left endpoint of $e$ lies on the arc $p q$. The area of the circular cap determined by the chord $e \cap \operatorname{disk}_{1.5}(s)$ in $\operatorname{disk}_{1.5}(s)$ is referred to as the outer loss. Denote by $\ell_{1}$ the constant

$$
\frac{9}{8}\left(\frac{\pi}{3}-2 \angle o p s-\sin \left(\frac{\pi}{3}-2 \angle o p s\right)\right) \approx 1.7915347 \times 10^{-3} .
$$

By Lemma 5, the outer loss is at most $\ell_{1}$. Let $e_{1}$ be the side of $P$ between $u_{1}$ and the adjacent outer vertex, $\varphi$ be the central angle of $e_{1}$, and $\delta$ be the central angle of $p u_{1}$. Denote by $x$ the intersection point between $e_{1}$ and $\operatorname{circle}_{1.5}(s)$. Since the right endpoint of $e_{1}$ is on the arc $p q$, we have $\delta<\varphi \leq \delta+\angle p o q$. We consider two cases according to whether $\delta$ is at least $3^{\circ}$ or not.

Case 1: $\delta \geq 3^{\circ}$. Let $v$ be the point on $\operatorname{circle}_{0.5}(o)$ at which $u^{\prime} u_{1}$ is tangent to circle $_{0.5}(o)$ (see Fig. 6). The area of the arc triangle surrounded by $e_{1}$, the ray $u_{1} v$ and $\operatorname{circle}_{1.5}(s)$ is referred to as the inner gain. Clearly, $\left|P \cap \operatorname{disk}_{1.5}(s)\right|$ is at least

Fig. 6 Inner gain

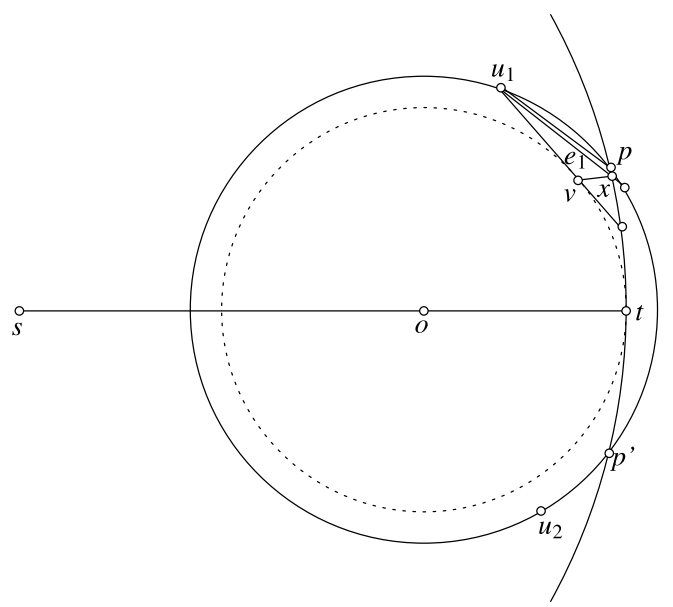


$\left|P^{\prime} \cap \operatorname{disk}_{1.5}(s)\right|$ plus the inner gain and minus the outer loss. Since the inner gain is more than $\left|\triangle u_{1} v x\right|$, we have

$$
\left|P \cap \operatorname{disk}_{1.5}(s)\right| \geq\left|P^{\prime} \cap \operatorname{disk}_{1.5}(s)\right|+\left|\triangle u_{1} v x\right|-\ell_{1} .
$$

First, we show that if $\delta \in\left[3^{\circ}, 48^{\circ}\right]$ then $\left|\Delta u_{1} v x\right|>\ell_{1}$, from which we have

$$
\left|P \cap \operatorname{disk}_{1.5}(s)\right|>\left|P^{\prime} \cap \operatorname{disk}_{1.5}(s)\right| \geq \sigma
$$

by Corollary 1 and Lemma 4. Clearly, $u_{1} v=\frac{1}{2 \sqrt{3}}$. Since

$$
\begin{gathered}
u_{1} x \geq u_{1} p=\frac{2}{\sqrt{3}} \sin \frac{\delta}{2}, \\
\angle v u_{1} x \geq \angle v u_{1} q=\frac{1}{2}\left(\frac{\pi}{3}-\delta-\angle p o q\right),
\end{gathered}
$$

we have

$$
\begin{aligned}
\left|\triangle u_{1} v x\right| & =\frac{1}{2} u_{1} v \cdot u_{1} x \cdot \sin \angle v u_{1} x \\
& \geq \frac{1}{6} \sin \frac{\delta}{2} \cdot \sin \left(\frac{\pi}{6}-\frac{\angle p o q}{2}-\frac{\delta}{2}\right) \\
& =\frac{1}{12}\left[\cos \left(\frac{\pi}{6}-\frac{\angle p o q}{2}-\delta\right)-\cos \left(\frac{\pi}{6}-\frac{\angle p o q}{2}\right)\right] .
\end{aligned}
$$

Since $\frac{\pi}{6}-\frac{\angle p o q}{2} \approx 26.274^{\circ}$, the last expression above is concave with $\delta \in\left[3^{\circ}, 48^{\circ}\right]$ and its minimum over $\left[3^{\circ}, 48^{\circ}\right]$ is achieved at boundary. A simple calculation yields that its minimum is achieved at $\delta=3^{\circ}$ and is greater than $\ell_{1}$. Hence, $\left|\triangle u_{1} v x\right| \geq \ell_{1}$ when $\delta \in\left[3^{\circ}, 48^{\circ}\right]$.

Now, we show that if $\varphi \in\left[12^{\circ}, 57^{\circ}\right]$, then $\left|\Delta u_{1} v x\right| \geq \ell_{1}$, from which we have

$$
\left|P \cap \operatorname{disk}_{1.5}(s)\right|>\left|P^{\prime} \cap \operatorname{disk}_{1.5}(s)\right| \geq \sigma
$$

by Corollary 1 and Lemma 4 . Since

$$
\begin{aligned}
u_{1} x & \geq u_{1} p=\frac{2}{\sqrt{3}} \sin \frac{\delta}{2} \geq \frac{2}{\sqrt{3}} \sin \frac{\varphi-\angle p o q}{2}, \\
\angle v u_{1} x & =\frac{\pi}{6}-\frac{\varphi}{2},
\end{aligned}
$$

we have

$$
\begin{aligned}
\left|\triangle u_{1} v x\right| & =\frac{1}{2} u_{1} v \cdot u_{1} x \cdot \sin \angle v u_{1} x \\
& \geq \frac{1}{6} \sin \left(\frac{\varphi}{2}-\frac{\angle p o q}{2}\right) \sin \left(\frac{\pi}{6}-\frac{\varphi}{2}\right)
\end{aligned}
$$




$$
\begin{aligned}
& =\frac{1}{12}\left[\cos \left(\varphi-\frac{\pi}{6}-\frac{\angle p o q}{2}\right)-\cos \left(\frac{\pi}{6}-\frac{\angle p o q}{2}\right)\right] \\
& =\frac{1}{12}\left[\cos \left(\frac{\pi}{6}-\frac{\angle p o q}{2}-\left(\frac{\pi}{3}-\varphi\right)\right)-\cos \left(\frac{\pi}{6}-\frac{\angle p o q}{2}\right)\right] .
\end{aligned}
$$

Since $\frac{\pi}{3}-\varphi \in\left[3^{\circ}, 48^{\circ}\right]$ when $\varphi \in\left[12^{\circ}, 57^{\circ}\right]$, the above expression is also greater than $\ell_{1}$. Hence, $\left|\triangle u_{1} v x\right|>\ell_{1}$ when $\varphi \in\left[12^{\circ}, 57^{\circ}\right]$.

Next, we assume that $\delta \notin\left[3^{\circ}, 48^{\circ}\right]$ and $\varphi \notin\left[12^{\circ}, 57^{\circ}\right]$. Since $\delta \geq 3^{\circ}$, we have $\varphi>\delta>48^{\circ}$, which implies that $\varphi>57^{\circ}$. Hence, the base angle of $P^{\prime}$ is $\frac{\angle u_{1} o u_{2}}{2}-\frac{\pi}{6}$. Since

$$
\begin{aligned}
\frac{\angle u_{1} o u_{2}}{2}-\frac{\pi}{6} & \geq \frac{\delta+\angle p o p^{\prime}}{2}-\frac{\pi}{6}=\frac{\delta}{2}+\angle p o q \\
& \geq \frac{\varphi-\angle p o q}{2}+\angle p o q=\frac{\varphi+\angle p o q}{2} \geq 32^{\circ},
\end{aligned}
$$

by Corollary 1 and Lemma 4 we have

$$
\left|P^{\prime} \cap \operatorname{disk}_{1.5}(s)\right| \geq f\left(32^{\circ}\right)>\sigma+\ell_{1} .
$$

Consequently, $\left|P \cap \operatorname{disk}_{1.5}(s)\right|>\sigma$.

Case 2: $\delta<3^{\circ}$. Let $u_{3}$ be the inner vertex of $P$ adjacent to $u_{1}$. Then, $u_{1} u_{3}$ is tangent to circle $_{0.5}(o)$ at the midpoint, denoted by $v$, of $u_{1} u_{3}$. Denote by $\theta_{1}$ the angle $\angle$ vot. Then, $\theta_{1}=\angle p o t+\delta+\frac{\pi}{6}<\frac{\pi}{2}$. Let $u^{\prime \prime}$ be the point such that the two line segments $u_{3} u^{\prime \prime}$ and $u_{2} u^{\prime \prime}$ are both tangent to circle $_{0.5}(o)$. Then, $u^{\prime \prime}$ is on the right side of the vertical line through $t$, and $u_{1}$ is on the line segment $u_{3} u^{\prime \prime}$. Let $P^{\prime \prime}$ be the canonical polygon of $u^{\prime \prime}$. The base angle of $P^{\prime \prime}$ is $\frac{\angle u_{1} o u_{2}}{2}$, which is greater than $\frac{\angle p o p^{\prime}}{2}=\angle$ pot $\approx 37.453^{\circ}$. By Corollary 1 and Lemma $4,\left|P^{\prime \prime} \cap \operatorname{disk}_{1.5}(s)\right| \geq f\left(\frac{\pi}{3}\right)$. Let $w$ be the intersection point between $v u_{1}$ and circle $_{1.5}(s)$ (see Fig. 7). The area of the arc triangle $u_{1} w x$ surrounded by the arc $w x$ and the two line segments $u_{1} w$ and

Fig. 7 Inner loss

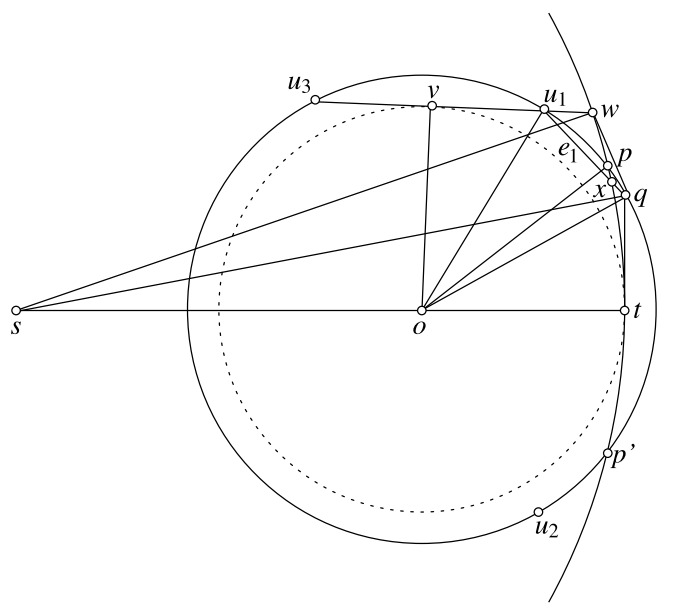


$u_{1} x$ is referred to as the inner loss and is denoted by $\ell_{2}$. Then,

$$
\left|P \cap \operatorname{disk}_{1.5}(s)\right| \geq\left|P^{\prime \prime} \cap \operatorname{disk}_{1.5}(s)\right|-\ell_{1}-\ell_{2} \geq f\left(\frac{\pi}{3}\right)-\ell_{1}-\ell_{2} .
$$

So, it is sufficient to show that $\ell_{2}<f\left(\frac{\pi}{3}\right)-\sigma-\ell_{1}$.

We first claim that $\ell_{2} \leq\left|\Delta u_{1} w q\right|$. Indeed, while $u_{1}$ is fixed the arc triangle $u_{1} w x$ grows when the right endpoint of $e_{1}$ moves toward $q$. Thus we only need to prove that the claim holds when the right endpoint of $e_{1}$ is $q$. Note that when $\theta_{1}<\frac{\pi}{2}$,

$$
\angle w s t<\arcsin \frac{1}{3}<2 \arcsin \frac{1}{3 \sqrt{3}}=2 \angle q s t .
$$

Thus, $\angle q s w=\angle w s t-\angle q s t<\angle q s t$. By the law of cosines, we have $q w<q t$. Hence,

$$
q s^{2}=s t^{2}+q t^{2}>s w^{2}+q w^{2},
$$

which implies that $\angle s w q$ is obtuse. So, the arc triangle $u_{1} w x$ is contained in $\Delta u_{1} w q$, and consequently the inner loss is at most $\left|\triangle u_{1} w q\right|$. Hence the claim holds. Therefore,

$$
\left|P \cap \operatorname{disk}_{1.5}(s)\right| \geq\left|P^{\prime \prime} \cap \operatorname{disk}_{1.5}(s)\right|-\left|\Delta u_{1} w q\right|-\ell_{1} \geq f\left(\frac{\pi}{3}\right)+\left|\Delta u_{1} v x\right|-\ell_{1} .
$$

Now, we claim that $\left|\Delta u_{1} w q\right|$ increases with $\theta_{1}$ and hence with $\delta$ when $\delta \leq 3^{\circ}$. Note that

$$
\left|\triangle u_{1} w q\right|=\frac{1}{2} u_{1} w \cdot u_{1} q \cdot \sin \angle q u_{1} w .
$$

Since ow increases with $\theta_{1}$ and

$$
u_{1} w=v w-\frac{1}{2 \sqrt{3}}=\sqrt{o w^{2}-1 / 4}-\frac{1}{2 \sqrt{3}},
$$

$u_{1} w$ increases with $\theta_{1}$. Since

$$
u_{1} q=\frac{2}{\sqrt{3}} \sin \left(\frac{\theta_{1}}{2}-\frac{\pi}{6}\right)
$$

$u_{1} q$ also increases with $\theta_{1}$. Since

$$
\angle q u_{1} w=\pi-\frac{\pi}{3}-\angle o u_{1} q=\frac{2 \pi}{3}-\frac{\pi-\angle q o u_{1}}{2}=\frac{\frac{\pi}{3}+\angle q o u_{1}}{2}=\frac{\theta_{1}}{2}
$$

$\angle q u_{1} w$ increases with $\theta_{1}$. Thus, our claim holds.

Denote by $\ell_{2}$ the area of $\Delta u_{1} w q$ when $\delta=3^{\circ}$. It is easy to verify that when $\delta=3^{\circ}$, $\theta_{1}=\angle p o t+\frac{11 \pi}{60}$ and

$$
\left|\triangle u_{1} w q\right|=\left(\frac{\sqrt{2}}{4} \sin \frac{\theta_{1}-\arccos \frac{1+2 \cos \theta_{1}}{3}}{2}-\frac{1}{12}\right)\left(\frac{\sqrt{3}}{2}-\cos \left(\theta_{1}-\frac{\pi}{6}\right)\right)
$$




$$
<f\left(\frac{\pi}{3}\right)-\sigma-\ell_{1}
$$

Therefore, $\ell_{2}<f\left(\frac{\pi}{3}\right)-\sigma-\ell_{1}$, which implies $\left|P \cap \operatorname{disk}_{1.5}(s)\right|>\sigma$.

\section{Independence Number vs. Connected Domination Number}

In this section, we present an improved upper bound on the independence number in terms of the connected domination number.

Theorem 2 Let $\alpha$ and $\gamma_{c}$ be the independence number and connected domination number of a connected UDG G. Then,

$$
\alpha \leq 3.4306 \gamma_{c}+4.8185 \text {. }
$$

We prove the above theorem by an integrated area and length argument. Let $U$ be a minimum CDS of $G$, and define

$$
\Omega=\bigcup_{u \in U} \operatorname{disk}_{1.5}(u)
$$

Consider a maximum independent set $I$ of $G$. We construct the Voronoi diagram defined by $I$. For each $o \in I$, we use $\operatorname{Vor}(o)$ to denote its Voronoi cell and call the set $\operatorname{Vor}(o) \cap \Omega$ as the truncated Voronoi cell of $o$. Clearly, $|\Omega|$ is the total area of truncated Voronoi cells of all nodes in $I$. We partition $I$ into two subsets $I_{1}$ and $I_{2}$ defined by

$$
\begin{aligned}
& I_{1}=\left\{o \in I: \operatorname{disk}_{1 / \sqrt{3}}(o) \subset \Omega\right\}, \\
& I_{2}=I \backslash I_{1} .
\end{aligned}
$$

Denote by $\alpha_{1}$ and $\alpha_{2}$ the size of $I_{1}$ and $I_{2}$ respectively. The next lemma provides a lower bound on each truncated Voronoi cell.

Lemma 6 For each o in $I_{1}$ (respectively, $I_{2}$ ), the area of its truncated Voronoi cell is at least $\sqrt{3} / 2$ (respectively, $\sigma$ ).

Proof Since the pairwise distances of the points in $I$ are at least one, the distance between $o$ and each side of $\operatorname{Vor}(o)$ is at least 0.5 and consequently $\operatorname{dis}_{0.5}(o) \subset \operatorname{Vor}(o)$. Next, we show that no vertex of $\operatorname{Vor}(o)$ is inside $\operatorname{disk}_{1 / \sqrt{3}}(o)$. Let $v$ be a vertex of $\operatorname{Vor}(o)$, and $e_{1}$ and $e_{2}$ be the two sides of $\operatorname{Vor}(o)$ incident to $v$ (see Fig. 8). Let $o_{1}$ (respectively, $o_{2}$ ) be the point which is symmetric to $o$ with respect to $e_{1}$ (respectively, $e_{2}$ ). Then, both $o_{1}$ and $o_{2}$ belong to $I$, and hence the three sides of $\triangle o_{1} o_{2}$ are all at least 1. Clearly, $v$ is the center of $\triangle o_{1} o_{2}$. Since at least one of the three central angles of $\triangle o o_{1} o_{2}$ is at most $120^{\circ}$, the circumscribing radius of $\triangle o o_{1} o_{2}$ is at least $1 / \sqrt{3}$. Thus, $o v \geq 1 / \sqrt{3}$.

Let $s$ be the node in the MCDS $U$ closest to $o$. Then, $o \in \operatorname{disk}_{1}(s)$. If $\operatorname{disk}_{1 / \sqrt{3}}(o) \subseteq$ $\operatorname{Vor}(o)$, then $|\operatorname{Vor}(o) \cap \Omega| \geq\left|\operatorname{disk}_{1 / \sqrt{3}}(o) \cap \operatorname{disk}_{1.5}(s)\right|>\sqrt{3} / 2$. So, we assume 
Fig. 8 Any vertex of $\operatorname{Vor}(o)$ is apart from $o$ by at least $1 / \sqrt{3}$



Fig. 9 Inserting operations

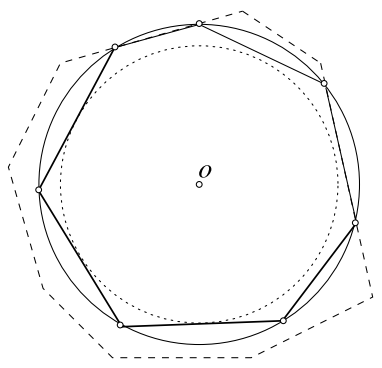

$\operatorname{disk}_{1 / \sqrt{3}}(o)$ is not fully contained in $\operatorname{Vor}(o)$. Then $\operatorname{Vor}(o)$ intersects $\operatorname{circle}_{1 / \sqrt{3}}(o)$. We construct a polygon $P \subseteq \operatorname{Vor}(o)$ satisfying that $P$ is inscribed in circle $_{1 / \sqrt{3}}(o)$ and $\operatorname{disk}_{0.5}(o) \subseteq P \subseteq \operatorname{Vor}(o)$. Let $Q$ be the sequence of intersecting points between $\operatorname{Vor}(o)$ and circle $_{1 / \sqrt{3}}(o)$ in the counterclockwise order. For each pair of successive $u$ and $v$ in $Q$, if $\angle u o v \leq \frac{\pi}{3}$, we add to $P$ a side between $u$ and $v$; otherwise, we add to $P$ a path inscribed in the arc from $u$ to $v$ satisfying that each edge in this path is either tangent to or disjoint from circle $_{1 / \sqrt{3}}(o)$ (see Fig. 9). The resulting polygon $P$ meets the requirement. By Theorem $1,|P| \geq \sqrt{3} / 2$.

If $o \in I_{1}$, then

$$
P \subseteq \operatorname{Vor}(o) \cap \operatorname{disk}_{1 / \sqrt{3}}(o) \subseteq \operatorname{Vor}(o) \cap \Omega,
$$

hence

$$
|\operatorname{Vor}(o) \cap \Omega| \geq|P| \geq \sqrt{3} / 2 .
$$

Now, we assume that $o \in I_{2}$. Note that $\left|P \cap \operatorname{disk}_{1.5}(s)\right|$ grows when moving $o$ away from $s$ along a fixed radius of $\operatorname{disk}_{1.5}(s)$. By Theorem $1,\left|P \cap \operatorname{disk}_{1.5}(s)\right| \geq \sigma$. Since

$$
P \cap \operatorname{disk}_{1.5}(s) \subseteq \operatorname{Vor}(o) \cap \Omega,
$$

we have

$$
|\operatorname{Vor}(o) \cap \Omega| \geq\left|P \cap \operatorname{disk}_{1.5}(s)\right| \geq \sigma .
$$

We define

$$
\Omega^{\prime}=\bigcup_{v \in U} \operatorname{disk}_{1.5-1 / \sqrt{3}}(v) .
$$

The next lemma gives an upper bound on the length of $\partial \Omega^{\prime}$. 
Lemma 7 The length of $\partial \Omega^{\prime}$ is at most $2(1-1 / \sqrt{3}) \alpha_{2}$.

Proof For each $o \in I_{2}$, let $o^{\prime}$ be a point in $U$ which is closest to $o$. Then,

$$
1.5-1 / \sqrt{3}<o o^{\prime} \leq 1 .
$$

Let $o^{\prime \prime}$ be the point which is the intersection of the segment $o o^{\prime}$ and circle $_{1.5-1 / \sqrt{3}}\left(o^{\prime}\right)$. Then, $o^{\prime \prime} \in \partial \Omega^{\prime}$ and $o o^{\prime \prime}=o o^{\prime}-o^{\prime} o^{\prime \prime} \leq 1-(1.5-1 / \sqrt{3})=1 / \sqrt{3}-0.5$. We call $o^{\prime \prime}$ the projection of $o$ on $\partial \Omega^{\prime}$. Consider two points $o_{1}$ and $o_{2}$ in $I$. Then,

$$
o_{1}^{\prime \prime} o_{2}^{\prime \prime} \geq o_{1} o_{2}-o_{1} o_{1}^{\prime \prime}-o_{2} o_{2}^{\prime \prime}>1-2(1 / \sqrt{3}-0.5)=2(1-1 / \sqrt{3}) .
$$

Now we decompose $\partial \Omega^{\prime}$ into disjoint arc-polygons, each of which is a maximally connected piece of $\partial \Omega^{\prime}$. We claim that if a piece contains $k \geq 1$ projections of points in $I_{2}$, then its length is at least $2(1-1 / \sqrt{3}) k$. Such a claim leads to the lemma immediately. The claim is true if $k \geq 2$. So we assume that $k=1$. Suppose that a piece $Q$ contains the projection $o^{\prime \prime}$ of a point $o \in I_{2}$ on $\partial \Omega^{\prime}$. Let $P$ be the region surrounded by the piece. Then exactly one of $o$ and $o^{\prime}$ is inside $P$. If $o^{\prime} \in P$, then the whole disk $\operatorname{disk}_{1.5-1 / \sqrt{3}}\left(o^{\prime}\right)$ is contained in $P$, and hence the length of $Q$ is at least $(3-2 / \sqrt{3}) \pi$, which is greater than $2(1-1 / \sqrt{3})$. So, we further assume that $o \in P$. We prove by contradiction that $P \varsubsetneqq \Omega$. Assume to the contrary that $P \subseteq \Omega$. Since $o \in I_{2}$, there is a point $p \in \operatorname{disk}_{1 / \sqrt{3}}(o) \backslash \Omega$. Clearly, $p \notin P$ and hence $o p$ intersects with $Q$. Let $q$ be an intersection point between $o p$ and $Q$, and $v$ be the center of the arc in $Q$ which contains $q$. Then, $v q=1.5-1 / \sqrt{3}$ and $p q \leq o p \leq 1 / \sqrt{3}$. Hence,

$$
v p \leq v q+p q \leq 1.5-1 / \sqrt{3}+1 / \sqrt{3}=1.5 \text {. }
$$

So, $p \in \operatorname{disk}_{1.5}(v) \subseteq \Omega$, which is a contradiction. Therefore, $P \varsubsetneqq \Omega$. Consider an arbitrary point $x \in P \backslash \Omega$. Then, the distance between $x$ and any point in $U$ is greater than 1.5, which implies that the distance between $x$ and any arc in $Q$ is more than $1.5-(1.5-1 / \sqrt{3})=1 / \sqrt{3}$. Hence. the disk $\operatorname{disk}_{1 / \sqrt{3}}(x)$ is contained in $P$, and as a result $|P|>\pi / 3$. By the well-known isodiametric inequality (see, e.g., in [8]), the length of $Q$ is more than $2 \pi / \sqrt{3}$, which is greater than $2(1-1 / \sqrt{3})$. Thus, the claim also holds when $k=1$.

By Lemma 6,

$$
|\Omega| \geq \frac{\sqrt{3}}{2} \alpha_{1}+\sigma \alpha_{2}=\frac{\sqrt{3}}{2} \alpha-\left(\frac{\sqrt{3}}{2}-\sigma\right) \alpha_{2},
$$

which implies

$$
\alpha \leq \frac{|\Omega|}{\frac{\sqrt{3}}{2}}+\left(1-\frac{\sigma}{\frac{\sqrt{3}}{2}}\right) \alpha_{2} .
$$


It is easy to prove by induction on $\gamma_{c}$ that

$$
|\Omega| \leq \frac{9}{2}\left(\left(\gamma_{c}-1\right)\left(\arcsin \frac{1}{3}+\frac{\sqrt{8}}{9}\right)+\frac{\pi}{2}\right),
$$

and the length of $\partial \Omega^{\prime}$ is at most

$$
2\left(3-\frac{2}{\sqrt{3}}\right)\left(\left(\gamma_{c}-1\right) \arcsin \frac{1}{3-\frac{2}{\sqrt{3}}}+\frac{\pi}{2}\right) .
$$

By Lemma 7,

$$
\begin{aligned}
\alpha_{2} \leq & \frac{2\left(3-\frac{2}{\sqrt{3}}\right)\left(\left(\gamma_{c}-1\right) \arcsin \frac{1}{3-\frac{2}{\sqrt{3}}}+\frac{\pi}{2}\right)}{2\left(1-\frac{1}{\sqrt{3}}\right)} \\
& =\frac{\sqrt{3}+7}{2}\left(\left(\gamma_{c}-1\right) \arcsin \frac{1}{3-\frac{2}{\sqrt{3}}}+\frac{\pi}{2}\right) .
\end{aligned}
$$

The three inequalities (1), (2) and (3) imply altogether that $\alpha$ is at most

$$
\begin{aligned}
& \left(\gamma_{c}-1\right)\left(\sqrt{27}\left(\arcsin \frac{1}{3}+\frac{\sqrt{8}}{9}\right)+\frac{\left(1-\frac{\sigma}{\frac{\sqrt{3}}{2}}\right)(\sqrt{3}+7)}{2} \arcsin \frac{1}{3-\frac{2}{\sqrt{3}}}\right) \\
& \quad+\frac{\pi}{2}\left(\sqrt{27}+\left(1-\frac{\sigma}{\frac{\sqrt{3}}{2}}\right) \frac{\sqrt{3}+7}{2}\right) \\
& \approx 3.4305176 \gamma_{c}+4.8184688 .
\end{aligned}
$$

Thus, Theorem 2 follows.

\section{Tighter Approximation Ratios}

In this section, we derive tighter bounds on the approximation ratio of the distributed algorithm proposed in [10] and the other greedy algorithm proposed in [11]. For the convenience of presentation, we call them WAF and WWY respectively. Let $G=(V, E)$ be a unit-disk graph. We denote by $\alpha$ and $\gamma_{c}$ the independence number and connected domination number of $G$ respectively. For any finite set $S$, we use $|S|$ to denote the cardinality of $S$.

The CDS produced by the algorithm WAF consists of a maximal independent set $I$ and a set $C$ of connectors. Specifically, let $T$ be an arbitrary rooted spanning tree of $G$. The set $I$ is selected in the first-fit manner in the breadth-first-search ordering in $T$ as follows. Initially $I$ is empty. For each node visited in the BFS ordering of $T$, it is added to $I$ if and only if it is not adjacent to any node in the current $I$. Let $s$ be 
a neighbor of the root of $T$ which is adjacent in $G$ to the most nodes in $I$. Then, $C$ consists of $s$ and the parents (in $T$ ) of the nodes in $I \backslash I(s)$. It was proved in [10] that $I \cup C$ is a CDS and $|I \cup C| \leq 8 \gamma_{c}-1$. Later on, two progressively improved tighter bounds $7.6 \gamma_{c}+1.4$ and $7 \frac{1}{3} \gamma_{c}$ were obtained in [12] and [11] respectively. The next theorem further improves the bound on $|I \cup C|$.

Theorem 3 The CDS produced by the algorithm WAF has size at most $6.862 \gamma_{c}+$ 8.637 .

Proof Let $I$ and $C$ be the set of nodes selected by the algorithm WAF in the first phase and the second phase respectively. Since $|C| \leq|I|-1$, we have

$$
|I \cup C| \leq 2|I|-1 \leq 2\left(3.4306 \gamma_{c}+4.8185\right)-1 \leq 6.862 \gamma_{c}+8.637 .
$$

So, the theorem follows.

In the next, we study the algorithm WWY. The first phase of this algorithm is the same as the algorithm WAF, and we let $I$ be the selected maximal independent set. But the second phase selects the connectors in a more economic way. For any subset $U \subseteq V \backslash I$, let $q(U)$ be the number of connected components in $G[I \cup U]$. For any $U \subseteq V \backslash I$ and any $w \in V \backslash I$, we define

$$
\triangle_{w} q(U)=q(U)-q(U \cup\{x\}) .
$$

The value $\triangle_{w} q(U)$ is referred to as the gain of $w$ with respect to $U$. The following lemma was proved in [11].

Lemma 8 Suppose that $q(U)>1$ for some $U \subseteq V \backslash I$. Then, there exists a $w \in$ $V \backslash(I \cup U)$ such that $\triangle_{w} q(U) \geq \max \left\{1,\left\lceil q(U) / \gamma_{c}\right\rceil-1\right\}$.

The second phase of the algorithm WWY runs as follows. We use $C$ to denote the sequence of selected connectors. Initially $C$ is empty. While $q(C)>1$, choose a node $w \in V \backslash(I \cup C)$ with maximum gain with respect to $C$ and add $w$ to $C$. When $q(C)=1$, then $I \cup C$ is a CDS. It was proved in [11] that $|I \cup C| \leq 6 \frac{7}{18} \gamma_{c}$. We derive a tighter bound on the output CDS in the theorem below.

Theorem 4 The CDS produced by the algorithm $\mathbf{W W Y}$ has size at most $6.075 \gamma_{c}+$ 5.425 .

Proof Let $I$ and $C$ be the set of nodes selected by the algorithm WWY in the first phase and the second phase respectively. If $\gamma_{c}=1$, then $|I| \leq 5$ and $|C| \leq 1$, hence $|I \cup C| \leq 6$. Thus, the theorem holds trivially if $\gamma_{c}=1$. If $|I| \leq 3 \gamma_{c}+2$, then $|I \cup C| \leq$ $2|I|-1 \leq 6 \gamma_{c}+3$, and the theorem also holds. From now on, we assume that $\gamma_{c} \geq 2$ and $|I|>3 \gamma_{c}+2$.

We break $C$ into three contiguous (and possibly empty) subsequences $C_{1}, C_{2}$ and $C_{3}$ as follows. $C_{1}$ is the shortest prefix of $C$ satisfying that $f\left(C_{1}\right) \leq 3 \gamma_{c}+2$, and 
$C_{1} \cup C_{2}$ is the shortest prefix of $C$ satisfying that $f\left(C_{1} \cup C_{2}\right) \leq 2 \gamma_{c}+1$. We will prove that

$$
\begin{aligned}
& \left|C_{1}\right| \leq \begin{cases}\frac{|I|}{3}-\gamma_{c}, & \text { if } f\left(C_{1}\right) \leq 3 \gamma_{c}+1, \\
\frac{|I|-2}{3}-\gamma_{c}, & \text { if } f\left(C_{1}\right)=3 \gamma_{c}+2 ;\end{cases} \\
& \left|C_{2}\right| \leq \begin{cases}\frac{\gamma_{c}}{2}, & \text { if } f\left(C_{1}\right) \leq 3 \gamma_{c}+1, \\
\frac{\gamma_{c}+1}{2}, & \text { if } f\left(C_{1}\right)=3 \gamma_{c}+2 ;\end{cases} \\
& \left|C_{3}\right| \leq 2 \gamma_{c}-1 .
\end{aligned}
$$

From the first two inequalities, we have

$$
\left|C_{1} \cup C_{2}\right| \leq \frac{|I|}{3}-\frac{\gamma_{c}}{2} .
$$

Using the third inequality, we have

$$
|C| \leq \frac{|I|}{3}-\frac{\gamma_{c}}{2}+2 \gamma_{c}-1=\frac{|I|}{3}+\frac{3}{2} \gamma_{c}-1 .
$$

So,

$$
\begin{aligned}
|I \cup C| & \leq \frac{4|I|}{3}+\frac{3}{2} \gamma_{c}-1 \leq \frac{4}{3}\left(3.4306 \gamma_{c}+4.8185\right)+\frac{3}{2} \gamma_{c}-1 \\
& \leq 6.075 \gamma_{c}+5.425 .
\end{aligned}
$$

Thus, the theorem follows.

First, we prove the first inequality. This inequality holds trivially if $C_{1}=\emptyset$. So we assume that $C_{1} \neq \varnothing$ and let $u$ be the last node in $C_{1}$. Then,

$$
f\left(C_{1} \backslash\{u\}\right) \geq 3 \gamma_{c}+3 .
$$

By Lemma 8, each node in $C_{1}$ has gain at least three. We consider two cases:

Case 1: $f\left(C_{1}\right) \leq 3 \gamma_{c}+1$. Then,

$$
3\left(\left|C_{1}\right|-1\right) \leq|I|-f\left(C_{1} \backslash\{u\}\right) \leq|I|-\left(3 \gamma_{c}+3\right),
$$

which implies $\left|C_{1}\right| \leq \frac{|I|}{3}-\gamma_{c}$.

Case 2: $f\left(C_{1}\right)=3 \gamma_{c}+2$. Then,

$$
3\left|C_{1}\right| \leq|I|-f\left(C_{1}\right)=|I|-\left(3 \gamma_{c}+2\right),
$$

which implies $\left|C_{1}\right| \leq \frac{|I|-2}{3}-\gamma_{c}$.

Now, we prove the second inequality. This inequality holds trivially if $\left|C_{2}\right| \leq 1$. So, we assume that $\left|C_{2}\right| \geq 2$ and let $v$ be the last node in $C_{2}$. Then,

$$
f\left(C_{1} \cup C_{2} \backslash\{v\}\right) \geq 2 \gamma_{c}+2 .
$$

By Lemma 8, each node in $C_{2}$ has gain at least two. We consider three cases. 
Case 1: $f\left(C_{1}\right) \leq 3 \gamma_{c}$. Then

$$
2\left(\left|C_{2}\right|-1\right) \leq f\left(C_{1}\right)-f\left(C_{1} \cup C_{2} \backslash\{v\}\right) \leq 3 \gamma_{c}-\left(2 \gamma_{c}+2\right),
$$

which implies that $\left|C_{2}\right| \leq \gamma_{c} / 2$.

Case 2: $f\left(C_{1}\right)=3 \gamma_{c}+1$. By Lemma 8, the first node in $C_{2}$ has gain at least three. Thus,

$$
3+2\left(\left|C_{2}\right|-2\right) \leq f\left(C_{1}\right)-f\left(C_{1} \cup C_{2} \backslash\{v\}\right) \leq 3 \gamma_{c}+1-\left(2 \gamma_{c}+2\right),
$$

which also implies that $\left|C_{2}\right| \leq \gamma_{c} / 2$. Therefore, $\left|C_{2}\right| \leq \gamma_{c} / 2$.

Case 3: $f\left(C_{1}\right)=3 \gamma_{c}+2$. By Lemma 8, the first node in $C_{2}$ has gain at least three. Thus,

$$
3+2\left(\left|C_{2}\right|-2\right) \leq f\left(C_{1}\right)-f\left(C_{1} \cup C_{2} \backslash\{v\}\right) \leq 3 \gamma_{c}+2-\left(2 \gamma_{c}+2\right),
$$

which implies $\left|C_{2}\right| \leq \frac{\gamma_{c}+1}{2}$.

Finally, we prove $\left|C_{3}\right| \leq 2 \gamma_{c}-1$. By Lemma 8 each node in $C_{3}$ has gain at least one.

Case 1: $f\left(C_{1} \cup C_{2}\right) \leq 2 \gamma_{c}$. Then

$$
\left|C_{3}\right| \leq f\left(C_{1} \cup C_{2}\right)-1 \leq 2 \gamma_{c}-1
$$

Case 2: $f\left(C_{1} \cup C_{2}\right)=2 \gamma_{c}+1$. By Lemma 8, the first node in $C_{3}$ has gain at least two. Thus,

$$
2+\left(\left|C_{3}\right|-1\right) \leq f\left(C_{1} \cup C_{2}\right)-1=2 \gamma_{c}+1-1,
$$

which implies that $\left|C_{3}\right| \leq 2 \gamma_{c}-1$.

\section{Discussions}

In this paper, we obtained a tighter relation between the independence number and connected domination number of a connected UDG. We actually proved the following stronger result on packing. Let $V$ be a set of $n$ nodes of a connected dominating set, and $\Gamma$ be the unions of unit-disks centered at $V$. Then, we can pack in $\Gamma$ at most $3.4306 n+4.8185$ points whose pairwise distances are greater than or equal to one. We'd like to emphasize that here we allow two points packed in $\Gamma$ to have distance equal to one. On the other hand, a packing of $3 n+3$ points in $\Gamma$ whose pairwise distances are greater than one was presented in [11]. It was also conjectured $3 n+3$ is the exact bound. Thus, there is still a gap between the bound $3.4306 n+4.8185$ derived in this paper and the conjectured bound $3 n+3$.

Acknowledgements The work described in this paper was partially supported by a grant from City University of Hong Kong (Project No. 7002611). Frances Yao was partially supported by the National Basic Research Program of China Grant 2007CB807900, 2007CB807901. Peng-Jun Wan was supported in part by National Science Foundation of USA under grants CNS-0831831 and CNS-0916666. 


\section{References}

1. Alzoubi, K.M., Wan, P.-J., Frieder, O.: Message-optimal connected dominating sets in mobile ad hoc networks. In: Proceedings of the 3rd ACM International Symposium on Mobile Ad Hoc Networking and Computing (MOBIHOC02), pp. 157-164 (2002)

2. Bharghavan, V., Das, B.: Routing in ad hoc networks using minimum connected dominating sets. In: Proceedings of the IEEE International Conference on Communications (ICC97), pp. 376-380 (1997)

3. Blum, J., Ding, M., Cheng, X.: Applications of connected dominating sets in wireless networks. In: Du, D.-Z., Pardalos, P. (eds.) Handbook of Combinatorial Optimization, pp. 329-369. Kluwer Academic, Dordrecht (2004)

4. Cadei, M., Cheng, X., Du, D.-Z.: Connected domination in ad hoc wireless networks. In: Proceedings of the 6th Joint Conference on Information Science (JCIS02), pp. 251-255 (2002)

5. Chvátal, V.: A greedy heuristic for the set-covering problem. Math. Oper. Res. 4(3), 233-235 (1979)

6. Funke, S., Kesselman, A., Meyer, U., Segal, M.: A simple improved distributed algorithm for minimum CDS in unit disk graphs. ACM Trans. Sens. Netw. 2(3), 444-453 (2006)

7. Li, Y.S., Thai, M.T., Wang, F., Yi, C.-W., Wan, P.-J., Du, D.-Z.: On greedy construction of connected dominating sets in wireless networks. Wirel. Commun. Mob. Comput. 5(8), 927-932 (2005)

8. Lin, F., Yang, X.: Geometric Measure Theory: An Introduction. International Press, Somerville (2003)

9. Stojmenovic, I., Seddigh, M., Zunic, J.: Dominating sets and neighbor elimination based broadcasting algorithms in wireless networks. IEEE Trans. Parallel Distrib. Syst. 13(1), 14-25 (2002)

10. Wan, P.-J., Alzoubi, K.M., Frieder, O.: Distributed construction of connected dominating set in wireless ad hoc networks. Mob. Netw. Appl. 9(2), 141-149 (2004)

11. Wan, P.-J., Wang, L., Yao, F.: Two-phased approximation algorithms for minimum CDS in wireless ad hoc networks. In: Proceedings of the 28th International Conference on Distributed Computing Systems, pp. 337-344 (2008)

12. Wu, W., Du, H., Jia, X., Li, Y., Huang, S.C.-H.: Minimum connected dominating sets and maximal independent sets in unit disk graphs. Theor. Comput. Sci. 352(1-3), 1-7 (2006) 